\title{
Role of interleukin 5-induced eosinophils in interleukin 33-triggered airway inflammation in mice
}

\author{
Hitomi Tanaka, ${ }^{1}$ Shigeki Katoh, ${ }^{1}$ Kazuko Uno, ${ }^{2}$ Toru Oga ${ }^{1}$
}

\begin{abstract}
Background: Interleukin (IL)-5 is essential for allergen induced eosinophilic airway inflammation, but not activation of T helper type 2 (Th2) cells in the lung. Although an excessive Th2 reaction is observed without IL-5 signaling, the mechanisms have remained unknown.
\end{abstract}

Objective: To evaluate the negative-feedback mechanism in eosinophilic airway inflammation, we examined IL-33 triggered eosinophilic airway inflammation in IL-5 $\mathrm{Ra}^{-/-}$mice.

Methods: Mice were administered intranasal IL-33 for 3 days. Airway hyperresponsiveness (AHR) was evaluated and bronchoalveolar lavage (BAL) was performed $24 \mathrm{~h}$ after the last IL-33 treatment. The number of inflammatory cells and cytokine levels in the BAL fluid (BALF) were analyzed, and histologic examination was performed.

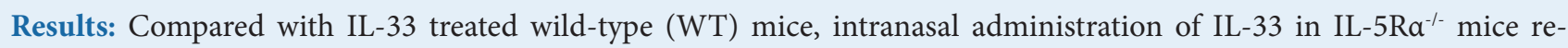
duced eosinophilic airway inflammation, AHR, and basement membrane thickening, while we found excessive IL-33 induced IL-5 and IL-13 production in the airway without IL-5 signaling. The numbers of eosinophils with a ringshaped nucleus (resident) and segmented nucleus (inflammatory) were increased in WT mice, but not in IL-5Ra ${ }^{-/-}$mice following intranasal administration of IL-33, and the numbers of SiglecF-positive eosinophils with (resident) or without (inflammatory) expression of CD62L were also significantly increased by IL-33 treatment in WT mice, but not in IL$5 \mathrm{Ra}^{-1-}$ mice. The number of ILC2 cells in the BALF was significantly higher in IL-33 treated IL-5R $\alpha^{-1-}$ mice than in IL-33 treated WT mice.

Conclusion: These findings suggest the possibility that IL-5 induced eosinophils contribute to the negative-feedback mechanisms in IL-33 induced ILC2 mediated airway inflammation.

Key words: IL-5Ra ${ }^{-/-}$mice, IL-33, airway inflammation, eosinophil, ILC2

\footnotetext{
Affiliations:

${ }^{1}$ Department of Respiratory Medicine, Kawasaki Medical School, Okayama, Japan

${ }^{2}$ Louis Pasteur Center for Medical Research, Kyoto, Japan
}

\author{
Corresponding author: \\ Shigeki Katoh \\ Department of Respiratory Medicine, Kawasaki Medical School \\ 577 Matsushima, Kurashiki, Okayama 701-0192, Japan \\ E-mail: kshigeki@med.kawasaki-m.ac.jp
}

\section{Introduction}

Asthma is a common respiratory disease characterized by airway hyperresponsiveness (AHR) and eosinophilic airway inflammation. Eosinophils are thought to be one of the most important effector cells in the development of asthma. ${ }^{1,2}$ Interleukin (IL)-5 plays an important role in the maturation, proliferation, and activation of eosinophils. ${ }^{3}$ We previously reported that IL-5 is involved in the pathogenesis of the allergic-asthma mouse model using IL-5 $\mathrm{Ra}^{-1-}$ mice. ${ }^{4,5}$ Several biologics targeted for IL-5/IL-5R are now available for the treatment of patients with severe eosinophilic asthma. ${ }^{6}$
Our previous findings demonstrated that IL-5 signaling is essential for allergen-induced eosinophilic airway inflammation, but not activation of $\mathrm{T}$ helper type 2 (Th2) cells in the lung. Interestingly, the allergen-induced Th2 reaction was found to be higher in IL-5 $\mathrm{Ra}^{-/-}$mice compared with wild type (WT) mice in 2 different mouse models using different allergens. ${ }^{4,5}$ These findings suggest the existence of negative-feedback mechanisms in Th2-related eosinophilic airway inflammation. 
Two subsets of eosinophils were recently reported in the mouse lung. One subset found in normal mouse lung comprises resident eosinophils ( $\mathrm{rEos}$ ), Siglec $\mathrm{F}^{\text {int }} \mathrm{CD} 62 \mathrm{~L}^{+} \mathrm{CD} 101^{\text {low }}$ cells with a ring-shaped nucleus. Another subset comprises inflammatory eosinophils (iEos), Siglec $\mathrm{F}^{\text {high }} \mathrm{CD} 62 \mathrm{~L}^{-} \mathrm{CD} 101^{\text {high }}$ cells with a segmented nucleus. Gene expression analyses revealed a more regulatory profile for rEos than for iEos.?

IL-33 is a ligand for ST2, a member of the IL-1 receptor family. ${ }^{8}$ IL-33 is an important cytokine involved in type 2 (T2) immunity and allergic respiratory disease. Abundantly expressed in lung epithelial cells, IL-33 plays a critical role in both innate and acquired immune responses in the airway. ${ }^{9}$ Group 2 innate lymphoid cells (ILC2s) are a subset of leukocytes that exhibit a lymphoid morphology but lack lymphocyte lineage markers. ILC2s proliferate in the lung and produce a large quantity of type 2 cytokines, such as IL- 5 and IL-13, in response to IL-33. ${ }^{10,11}$ Previous experimental models demonstrated the importance of the IL-33/ILC2 axis in innate-type allergic airway inflammation. In vivo treatment with IL-33 induces IL-5 and IL-13 production in the lung, and eosinophilic airway inflammation without allergen exposure. Intranasal administration of IL-33 induces asthma-like symptoms, such as AHR and airway inflammation, even in the absence of $\mathrm{T}$ and $\mathrm{B}$ cells. ${ }^{12,13}$

In the present study, we characterized an IL-33-triggered non-allergic asthma-like mouse model, and evaluated the role of IL-5-induced eosinophils in the development of this model using IL-5 $\mathrm{Ra}^{-1-}$ mice. Further, we analyzed the T2 responses in this non-allergic asthmatic model without IL-5 signaling.

\section{Methods \\ Animal models}

C57BL/6 mice (female, 8-12 weeks old) obtained from Charles River Laboratory (Yokohama, Japan) were used in this study. IL-5 $\mathrm{Ra}^{-1-}$ mice on a C57BL/6 background ${ }^{5}$ were bred in the experimental animal center of Kawasaki Medical School. To produce a mouse model of non-allergic asthma, mice were treated by intranasal administration of IL-33 (40 $\mu \mathrm{l}$ of $0.5 \mu \mathrm{g}$, R\&D Systems, Minneapolis, MN, USA) for 3 days (WT-IL-33). For the control group, phosphate-buffered saline (PBS) was administered (WT-PBS). These groups were compared with regard to the following characteristics of bronchial asthma to confirm acquisition of the pathology: (1) eosinophilic inflammation of the airway, and (2) enhancement of airway sensitivity. In addition, 8- to 12 -week old female IL-5 $\mathrm{Ra}^{-1-}$ mice were similarly treated (IL-5 $\mathrm{Ra}^{-1-}$-IL-33, IL-5 $\mathrm{R}^{-1-}-\mathrm{PBS}$ ) and compared to investigate the involvement of IL-5 in the pathogenesis. Animals were anesthetized by inhalation of sevoflurane. After confirming adequate anesthesia, $40 \mu \mathrm{L}$ of $0.5 \mu \mathrm{g}$ IL-33 was administered at the same volume and schedule as the control group. All animal protocols and experiments in the present study were approved by the institutional animal care and use committee of Kawasaki Medical School (Protocol Numbers: 19-009).

\section{Airway hyperresponsiveness (AHR)}

To evaluate the AHR, mice were forced to inhale PBS or acetyl- $\beta$-methylcholine chloride $(3,6,12.5,25,50$, or $100 \mathrm{mg} /$ $\mathrm{mL}$; Millipore Sigma, St. Louis, MO, USA) for $3 \mathrm{~min}$. The sRaw was measured in awake mice using the 2-chambered, double-flow plethysmograph system (Pulmos; M.I.P.S, Osaka, Japan). The mice were allowed to rest for $1 \mathrm{~min}$ and then the sRaw was measured for $2 \mathrm{~min}$. AHR is expressed as the concentration of acetyl- $\beta$-methylcholine chloride required to increase sRaw 2-fold $\left(\mathrm{PC}_{200}\right)$, similar to the $\mathrm{PC}_{20}$ measure used for patients with asthma.

\section{Collection of blood, bronchoalveolar lavage fluid (BALF), and lung tissue}

The mice were killed by carbon dioxide inhalation. The blood and bronchoalveolar lavage fluid (BALF) were collected, and the lungs were removed for pathologic evaluation by hematoxylin and eosin (HE), direct fast scarlet (DFS), and Periodic acid-Schiff (PAS) staining. The mucus score and basement membrane thickness were estimated in PAS-stained lung. A total of 10 airways of 3 mice from each group were categorized according to the abundance of PAS goblet cells, and assigned numerical scores ( $0:<5 \%$ goblet cells; $1: 5-25 \%$; 2: $25-50 \%$; 3: 50-75\%; 4: > 75\%). The mean basement membrane thickness was measured in 10 airways of 3 mice from each group. Bronchoalveolar lavage (BAL) was performed 24 $\mathrm{h}$ after the last IL-33 (or PBS) administration, and BALF was obtained by washing the lungs with $4 \times 1 \mathrm{ml}$ of PBS and centrifuged. The supernatant of the first wash was stored at $-80^{\circ} \mathrm{C}$ until use. Cell pellets of all washes were collected and resuspended in $1 \mathrm{ml}$ PBS. The number of BALF cells was counted using a cell counter. Cytospin slides were stained with DiffQuik (Sysmex, Kobe, Japan). Differential cell counts were performed on at least 400 cells.

\section{Enzyme-linked immunosorbent assay (ELISA)}

Amounts of IL-5, IL-7, IL-13 (R\&D Systems), and eosinophil-derived neurotoxin (EDN; Aviva System Biology, San Diego, CA, USA) in the BALF were measured using an enzyme-linked immunosorbent assay (ELISA). The detection limits were $7.0 \mathrm{pg} / \mathrm{mL}$ for IL-5, $6.3 \mathrm{pg} / \mathrm{mL}$ for IL-7, $1.5 \mathrm{pg} / \mathrm{mL}$ for IL-13, and $39.4 \mathrm{pg} / \mathrm{mL}$ for EDN. Concentrations below the detection limits were assumed to be zero for statistical analysis.

\section{Cytokine and chemokine measurements.}

Cell-free BALF was analyzed for cytokine production. Concentrations of IL-2, IL-4, IL-9, IL-10, IL-17, eotaxin, granulocye colony stimulation factor (G-CSF), interferon (IFN)- $\gamma$, and monocyte chemoattractant protein (MCP)-1 were determined in a Bio-plex pro mouse cytokine standard 23-plex set (Bio-Rad, Richmond, CA). Concentrations of transforming growth factor (TGF)- $\beta_{1}$ were determined in a Bio-plex pro TGF- $\beta_{1}$ standard 3-plex set (Bio-Rad). Assays were performed according to the respective manufacturer's instructions. The detection limits were $3.72 \mathrm{pg} / \mathrm{mL}$ for IL-2, 6.98 $\mathrm{pg} / \mathrm{mL}$ for IL-4, $6.89 \mathrm{pg} / \mathrm{mL}$ for IL-9, $2.95 \mathrm{pg} / \mathrm{mL}$ for IL-10, 
$2.65 \mathrm{pg} / \mathrm{mL}$ for IL-17, $147.4 \mathrm{pg} / \mathrm{mL}$ for eotaxin, $5.1 \mathrm{pg} / \mathrm{mL}$ for G-CSF, $1.84 \mathrm{pg} / \mathrm{mL}$ for IFN- $\gamma, 22.4 \mathrm{pg} / \mathrm{mL}$ for MCP-1, and 3.9 $\mathrm{pg} / \mathrm{mL}$ for TGF- $\beta_{1}$. Concentrations below the detection limits were assumed to be zero for statistical analysis.

\section{Flow cytometry analyses of ILC2s and eosinophils}

Cells were stained with the following antibodies for ILC2s: PE-anti-mouse CD4, APC-anti-mouse CD25 were purchased from eBioscience (San Diego, CA, USA). PE/Cy7anti-mouse ST2 monoclonal antibodies, biotin-anti-mouse CD3, biotin-anti-mouse CD19, biotin-anti-mouse B220, biotin-anti-mouse CD11b, biotin-anti-mouse CD11c, biotin-anti-mouse Gr-1, biotin-anti-mouse NK1.1, streptavidin-FITC were purchased from BioLegend (San Diego, CA, USA). Cells were stained with the following antibodies for eosinophils: PE-anti-mouse CD125, PE/Cy7-anti-mouse CD62L, FITCanti-mouse F4/80, PerCP/Cy5.5-anti-mouse CCR3 were purchased from BioLegend (San Diego, CA, USA). APC/Cy7anti-mouse SiglecF, Alexa Fluor 647-anti-mouse CD101 were purchased from BD Biosciences (San Jose, CA, USA).

Staining reactions were performed at $4^{\circ} \mathrm{C}$ after incubation with 2.4G2 Fc receptor blocking antibody against CD16/32 (eBioscience, San Diego, CA, USA) to reduce non-specific binding. Cell phenotyping was performed on a FACSCanto II (BD Biosciences). FSC-W and FSC-A discrimination was used to exclude doublet cells, and Fixable Viability Dye eFluor $^{\mathrm{m}} 506$ (FVD506) (eBiosciences) was used to discriminate between dead and living cells. Results were analyzed with FlowJo software (Tree Star). Isotype antibody controls were used to develop the gating and data analysis strategies. For ILC2 staining, we carefully gated only small and non-granular cells by forward and side scatters, respectively, and excluded the large and highly granular BALF cells from analysis. Lineage-positive cells were excluded using specific antibodies such as: anti-mouse CD3, anti-mouse CD19, anti-mouse B220, anti-mouse CD11b, anti-mouse CD11c, anti-mouse Gr-1, and anti-mouse NK1.1. Lineage-negative cells were analyzed for CD25 and ST2 expression by flow cytometry. For eosinophil staining, we carefully gated medium and granular cells by forward and side scatters, respectively. SiglecF- positive granulocytes were identified eosinophils and analyzed for CD62L and CD101 expression.

\section{Statistical analysis}

All data are expressed as the mean \pm standard error (SEM). Statistical analysis was performed using GraphPad Prism version 8.4 for Windows (GraphPad Software, San Diego, CA, USA). Statistical analyses were performed by 2 -way ANOVA and the Mann-Whitney $U$ test for comparison values between 2 groups. Differences with probability values of less than 0.05 were considered significant.

\section{Results}

Evaluation of IL-33-triggered airway inflammation in IL$\mathbf{5} \boldsymbol{R} \boldsymbol{\alpha}^{-/-}$mice

To evaluate the IL-33-triggered airway inflammation, we measured the AHR to methacholine, and inflammatory cell numbers and cytokine concentrations in the BALF at $24 \mathrm{~h}$ after the final IL-33 challenge. Intranasal treatment of IL-33 induces AHR in both WT and IL-5 $\mathrm{Ra}^{-/}$mice. Interestingly, IL-33 induced AHR was significantly lower in IL-5 $\mathrm{Ra}^{-/}$mice than in WT mice $\left(\mathrm{Mch}^{\mathrm{PC}}{ }_{200} 34.1 \pm 1.67\right.$ vs. $18.3 \pm 1.80 \mathrm{mg} /$ $\mathrm{mL}, p$ value $<0.01$, Figure 1a). After IL-33 exposure, the numbers of inflammatory cells other than eosinophils in the BALF were increased in both WT mice and in IL-5 $\mathrm{Ra}^{-/-}$mice. The number of eosinophils in the BALF was increased in WT mice, but not in IL-5R $\alpha^{-1-}$ mice $(p<0.01$, Figure 1b). The numbers of neutrophils and macrophages were significantly higher in IL-33-treated IL-5 $\mathrm{Ra}^{-/-}$mice than in IL-33-treated WT mice $(p<0.01$, Figure 1b). Cytokine and chemokine levels were evaluated in the BALF. IL-5 and IL-13 levels were increased by IL-33 exposure in both WT and IL-5 $\mathrm{Ra}^{-/-}$mice. Surprisingly those levels were higher in IL-5 $\mathrm{Ra}^{-1-}$ mice compared with WT mice (IL-5: $954.1 \pm 79.4$ vs. $479.8 \pm 47.2 \mathrm{pg} /$ mL, IL-13: $4434 \pm 326.4$ vs. $1900 \pm 190.1 \mathrm{pg} / \mathrm{mL} p<0.01$, Figure 1c). Furthermore, levels of eotaxin, IL-17, G-CSF, and MCP-1 in the BALF were also higher in IL-5 $\mathrm{Ra}^{-1-}$ mice compared with WT mice $(p<0.01$, Figure 1c).

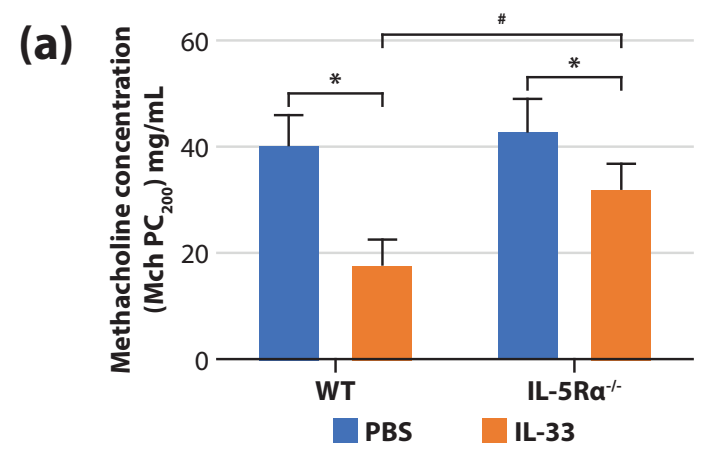

Figure 1. Effects of IL-5 on IL-33-triggered eosinophilic airway inflammation in mice.

Airway hyperresponsiveness (AHR) was measured and expressed as $\mathrm{PC}_{200}$ sRaw $(\mathrm{mg} / \mathrm{mL}$ ) as described in the Methods (a). Inflammatory cells in the BALF were counted. The details are described in the Methods (b). BALF IL-5 and IL-13 levels were measured by ELISA. Eotaxin, IL-17, G-CSF, and MCP-1 levels in the BALF were determined using Bio-Plex. ${ }^{*}$ Comparison between the IL-33 and PBS groups $(p<0.05)$. " Comparison between the WT and IL-5R ${ }^{-/-}$groups $(p<0.05)$. Values are presented as the mean \pm SEM from 6 mice per group. The significance of the differences was analyzed using 2-way ANOVA. 
(b)
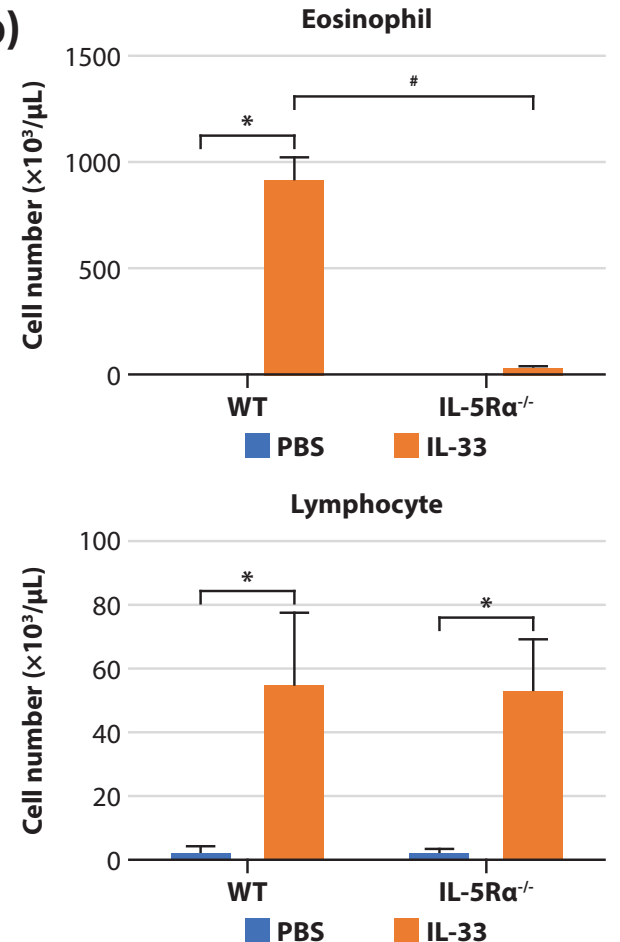
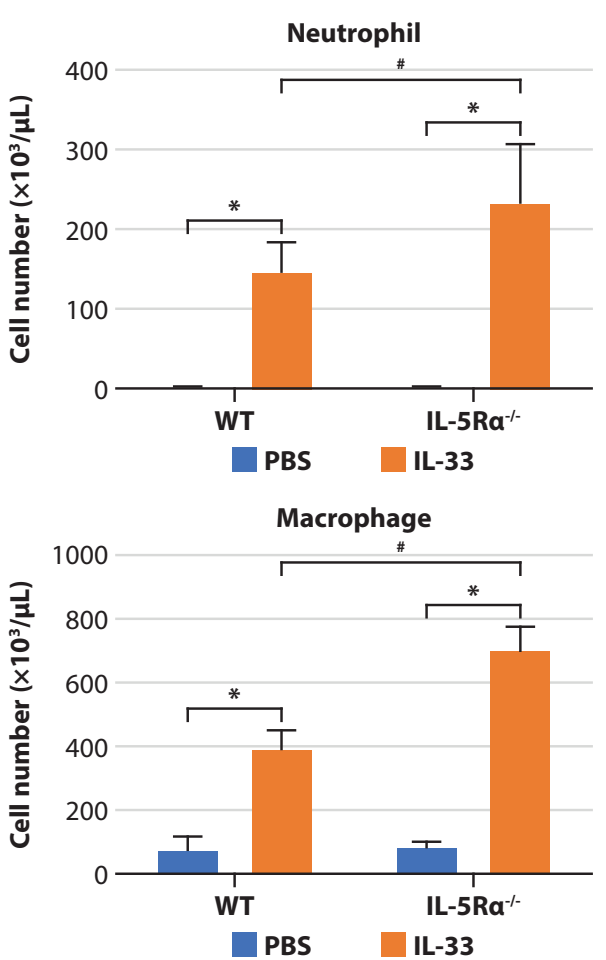

(c)

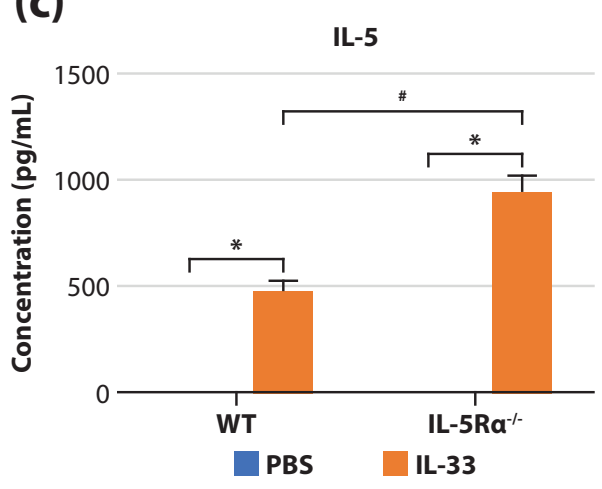

IL-17
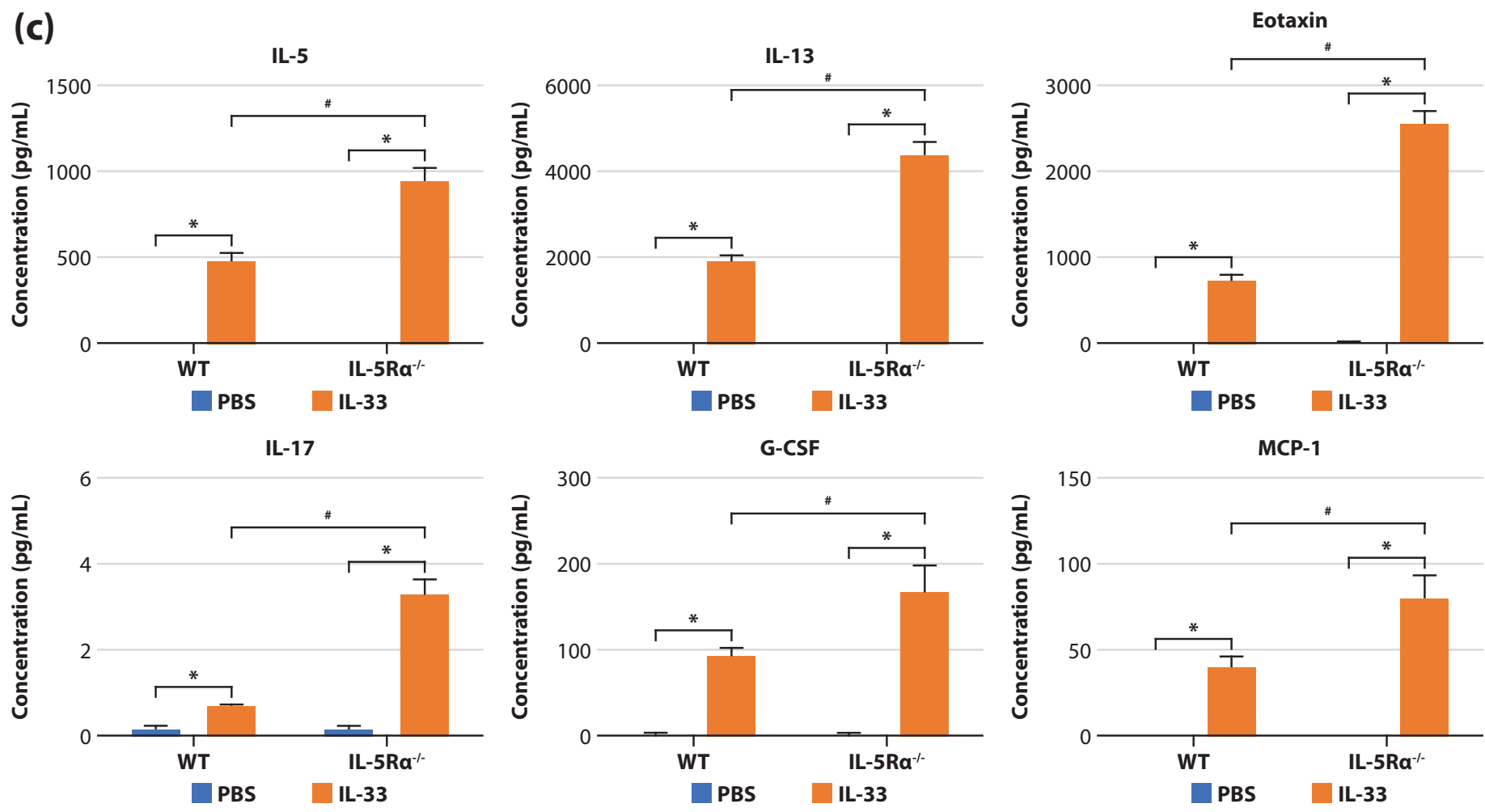

Figure 1. (Continued)

Histologic examination of IL33-triggered airway inflamma-

\section{tion in mice}

Similarly to the BALF findings, bronchial and lung perivascular inflammatory cell infiltration were induced by IL-33 in both WT and in IL-5 $\mathrm{Ra}^{-/-}$mice (Figure 2a), and eosinophil infiltration was observed in WT mice, but not in IL-5 $\mathrm{R}^{-1-}$ mice (Figure 2b). Further, to examine the involvement of IL-5-activated eosinophils in mucus secretion and basement membrane thickening in the airways, we evaluated PASstained lung sections. IL-33-induced mucus-secreting goblet cells were increased in both WT and IL-5 $\mathrm{Ra}^{-/-}$mice. Airway basement membrane thickness was induced by intranasal treatment of IL-33 in both WT and IL-5Ra ${ }^{-1-}$ mice. Basement membrane thickness, but not level of goblet cell hyperplasia was lower in IL-5 $\mathrm{Ra}^{-1}$ mice compared with WT mice $(p<$ 0.01, Figure 2c, 2d). 
(a)

WT

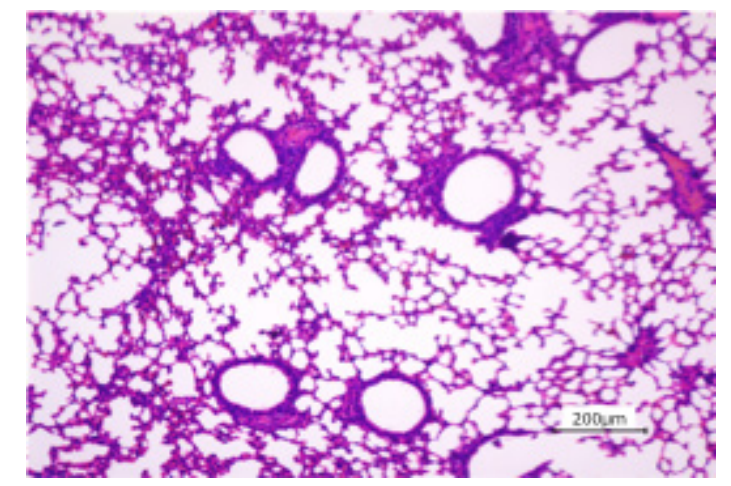

IL-33

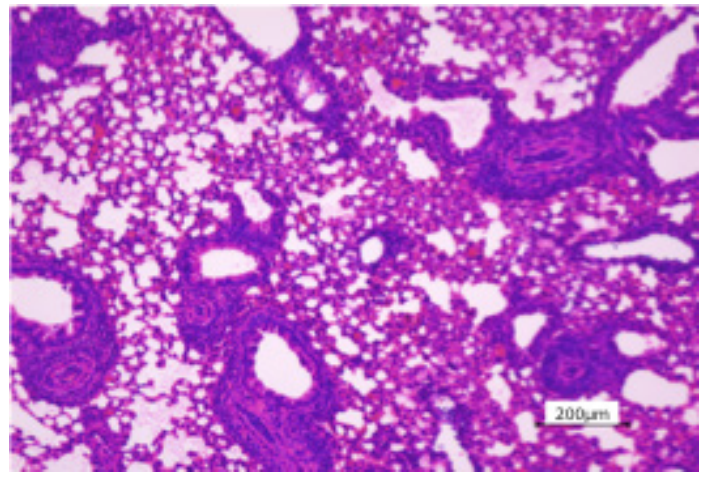

IL-5Ra $\mathbf{R}^{-1}$
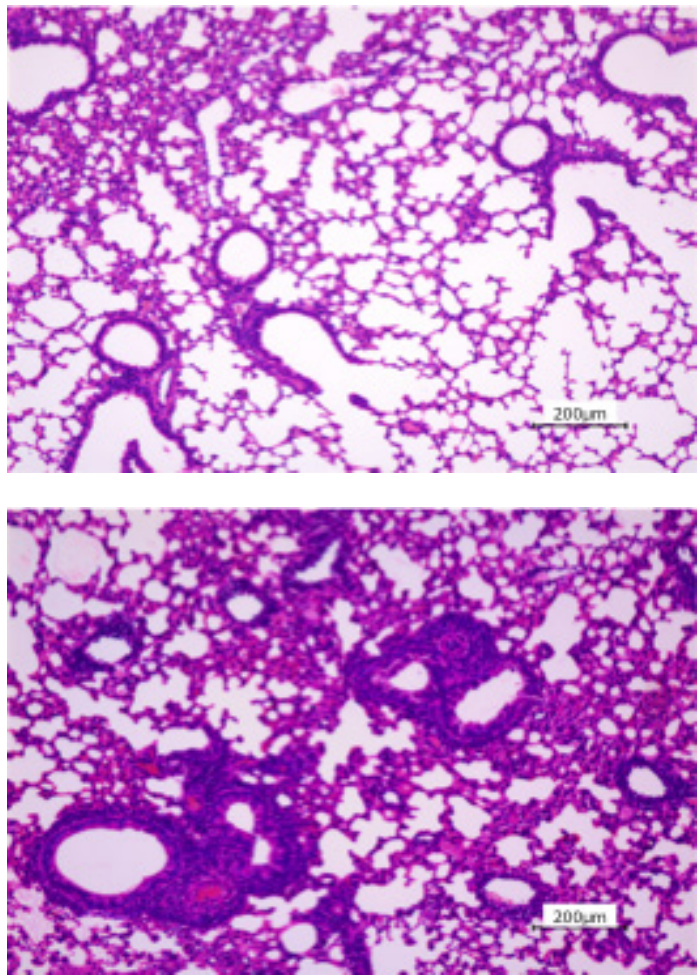

(b)

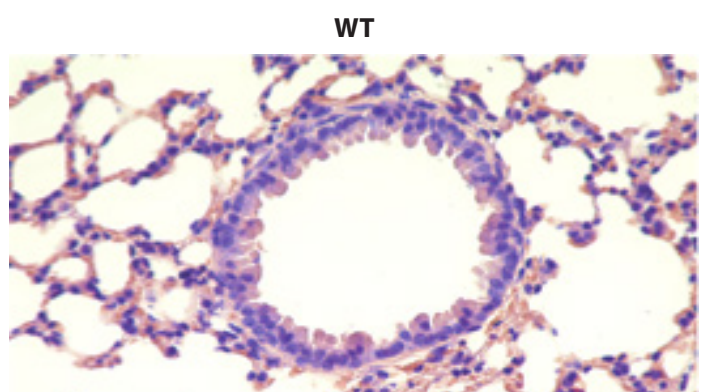

PBS
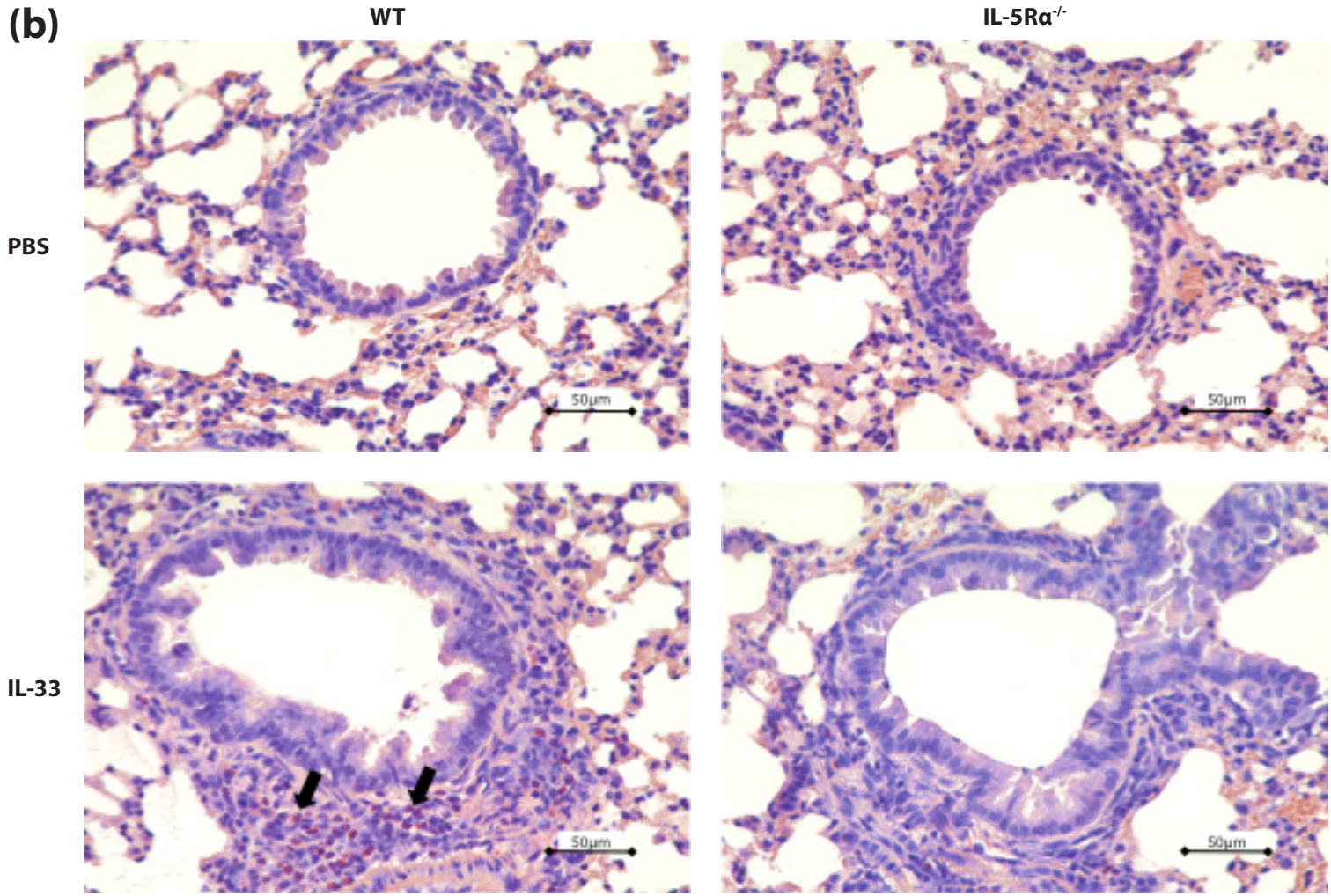

Figure 2. Histologic examination of IL-33-triggered eosinophilic airway inflammation in mice. Histologic examination of formalin-fixed lung sections stained with hematoxylin and eosin (original magnification: $\times 10)(a)$, Direct fast scarlet (DFS)-stained lung sections (original magnification: $\times 40$ ). Arrows indicate DFS-positive eosinophils in WT IL-33 mice (b). Periodic acid-Schiff $(\mathrm{PAS}$ )-stained lung sections (original magnification: $\times 40)(\mathrm{c})$. Mucus score and basement membrane thickening were determined as described in the Methods (d). A total of 10 airways of 3 mice from each group were analyzed. ${ }^{*}$ Comparison between the IL-33 and PBS groups $(p<0.05)$. "Comparison between the WT and IL-5R $\alpha^{-/}$groups $(p<0.05)$. The measurement results are presented as the mean \pm SEM in 3 mice. The significance of differences was analyzed using 2-way ANOVA. 
(c)

PBS
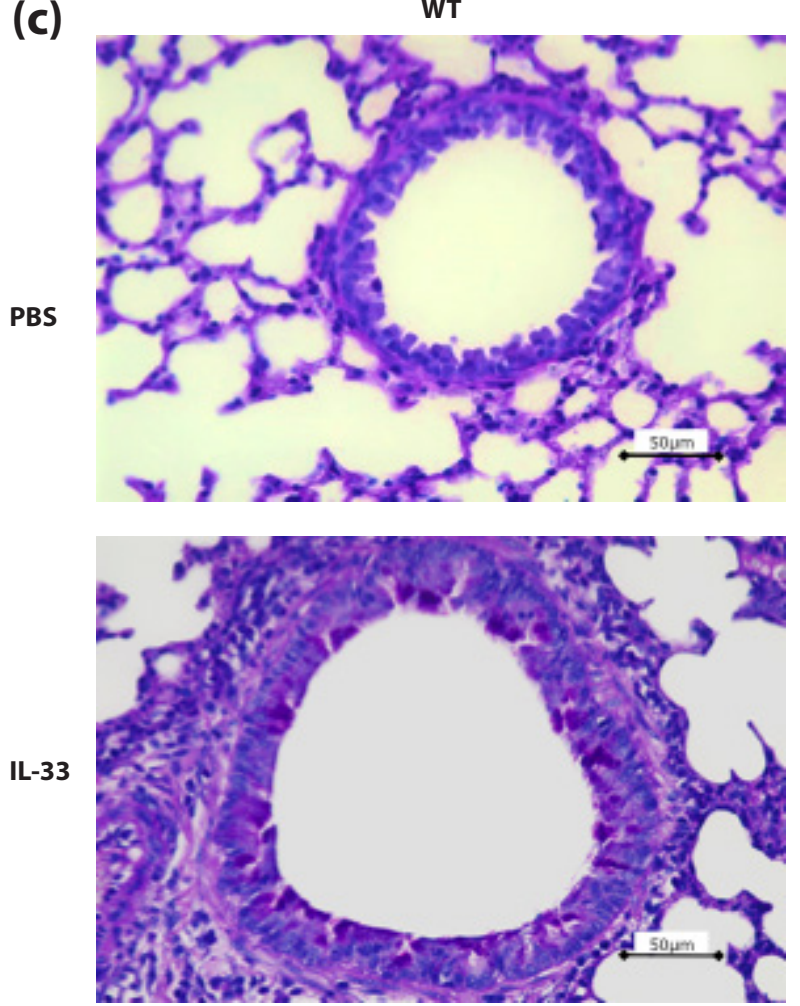

(d)

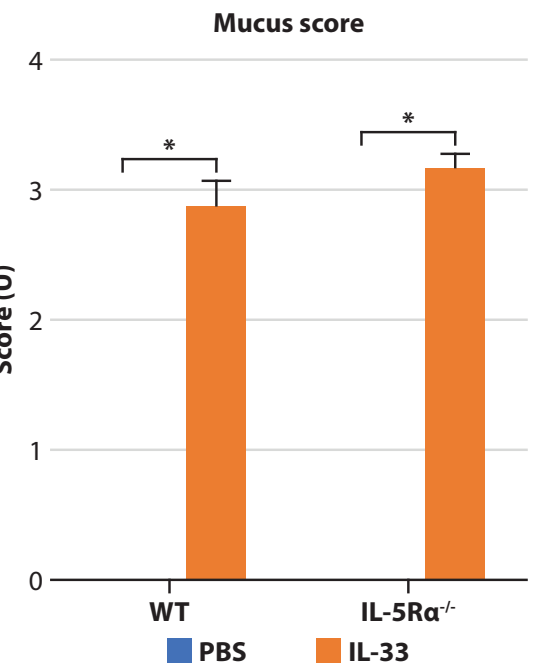

Figure 2. (Continued)

Evaluation of BALF eosinophils and ILC2s in IL-33-triggered airway inflammation

To investigate the factors involved in IL-33-induced AHR, we evaluated eosinophils by karyomorphism and cell surface markers and the concentration of eosinophil-derived neurotoxin in the BALF. The numbers of both the ring-shaped nucleus resident and segmented nucleus inflammatory eosinophils were increased in WT, but not in IL-5 $\mathrm{Ra}^{-/-}$mice (Figure 3a). The numbers of both the SiglecF-positive CD62L-negative inflammatory eosinophils and SiglecF-positive CD62L-positive resident eosinophils were also significantly increased by IL-33 treatment in WT mice, but not in IL-5 $\mathrm{Ra}^{-1-}$ mice (Figure $3 \mathbf{b}$ ). Further, the concentration of eosinophil-derived neurotoxin $(\mathrm{EDN})$ in the BALF was higher in IL-33 treated-WT mice than in IL-33-treated IL-5Ra ${ }^{-1-}$ mice $(p<0.01$, Figure $3 c)$.
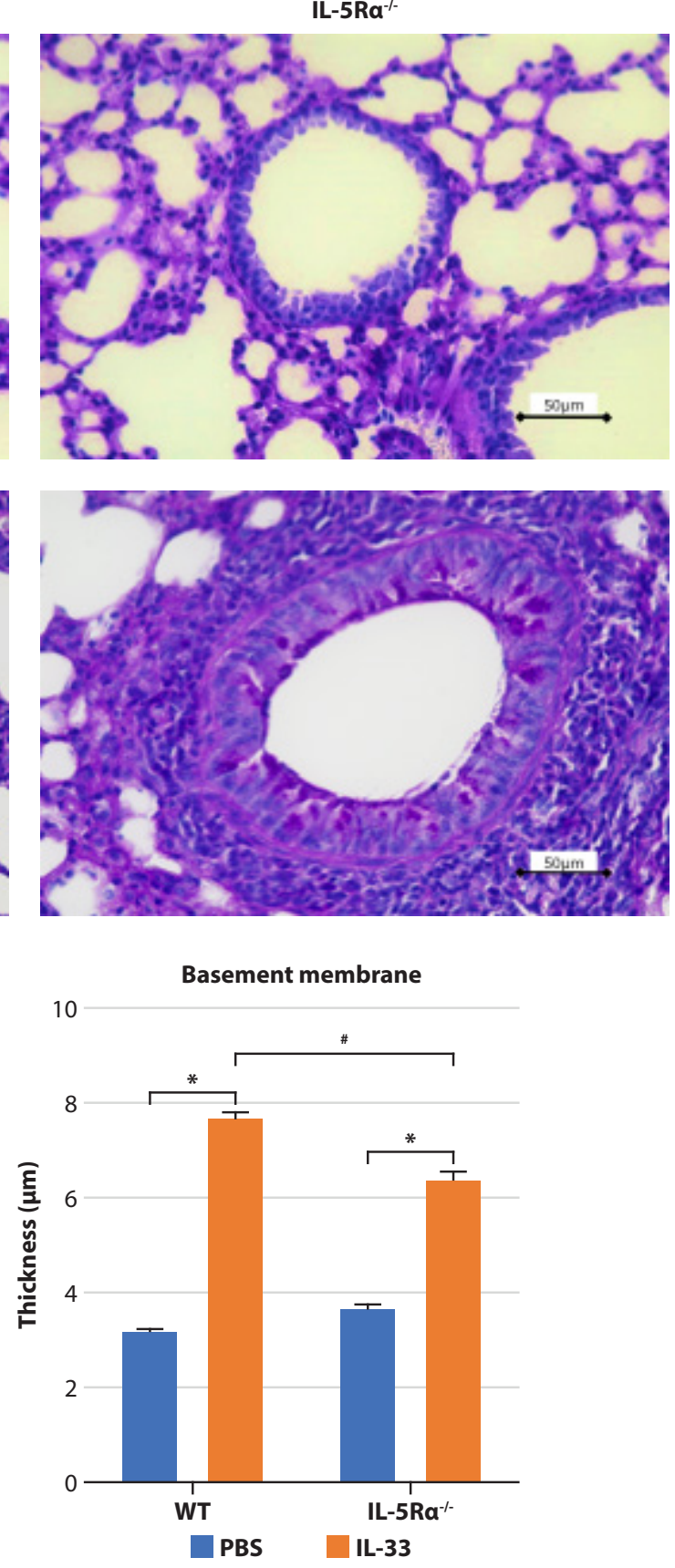

Finally, to examine the contribution of ILC2s to the IL-33induced eosinophilic airway inflammation, we analyzed ILC2s in the BALF by flow cytometry. Interestingly, the numbers of lineage-negative CD25-positive ILC2s in the BALF was significantly higher in IL-33-treated IL-5 $\mathrm{Ra}^{-/-}$mice than in IL-33treated WT mice (Figure 3d). We evaluated the contribution of the cytokines to the growth, activation, and suppression of ILC2s. ${ }^{14}$ BALF levels of stimulatory cytokines of ILC2s, such as IL-2, IL-4, and IL-7, were under the detection limit (data not shown). Following IL-33 treatment, the levels of suppressive cytokines of ILC2s, such as IL-10, IFN- $\gamma$, and TGF- $\beta_{1}$ in the BALF were significantly higher in IL-5 $\mathrm{Ra}^{-/}$mice compared with WT mice (Figure 3e). 
(a)

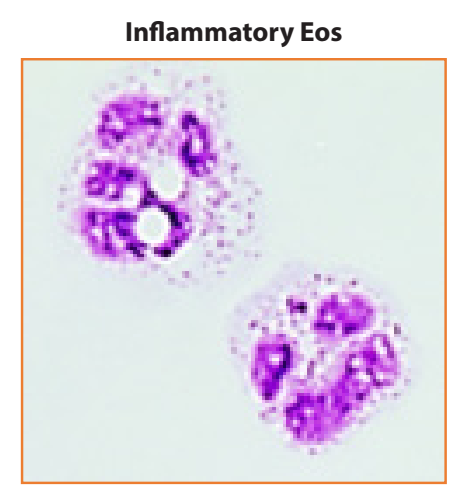

(b)
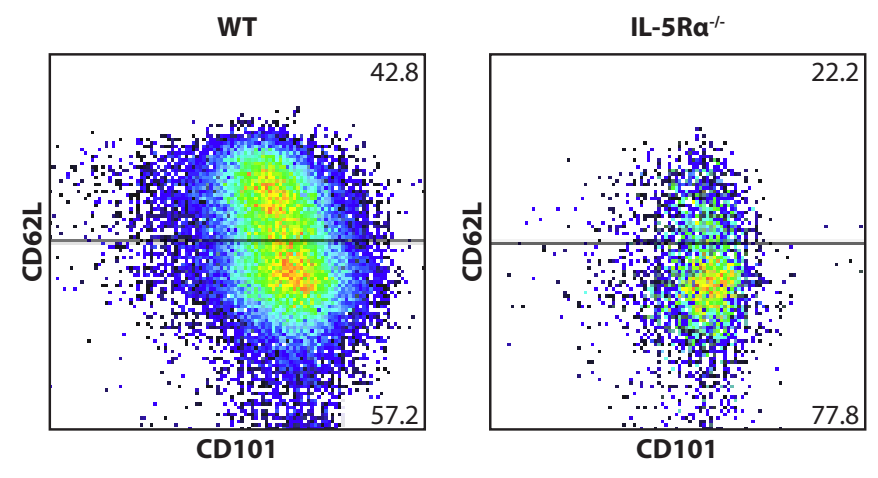

Karyomorphism
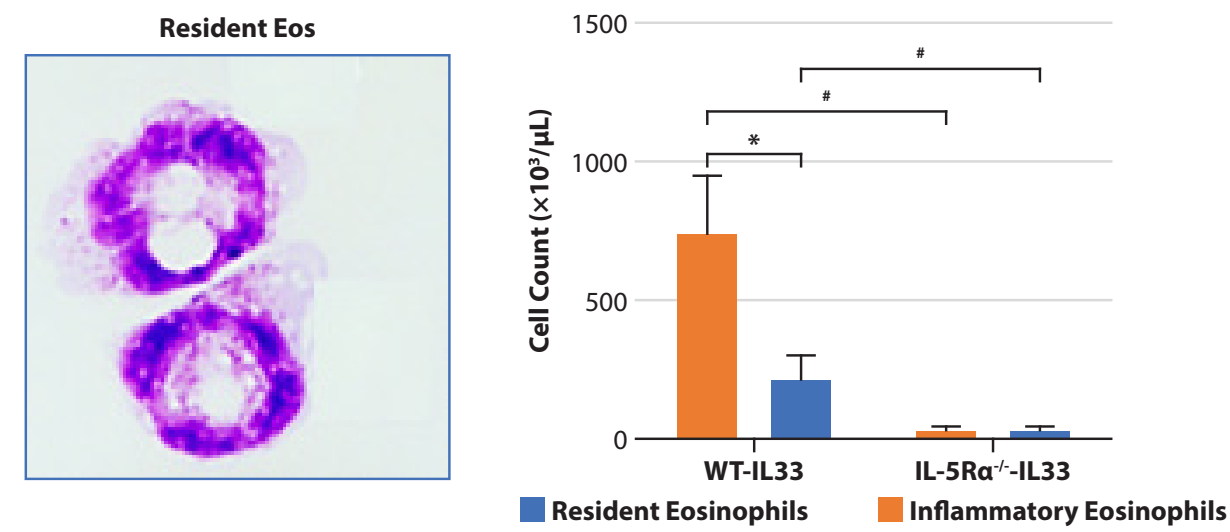

Flow cytometry

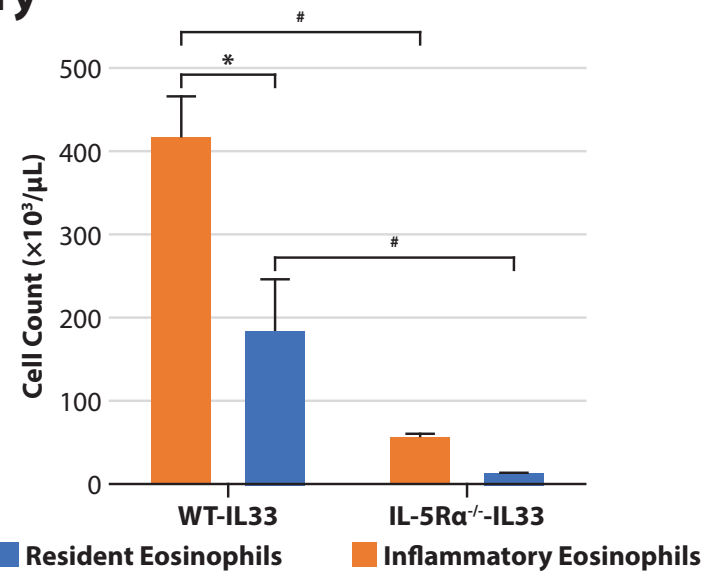

(c)

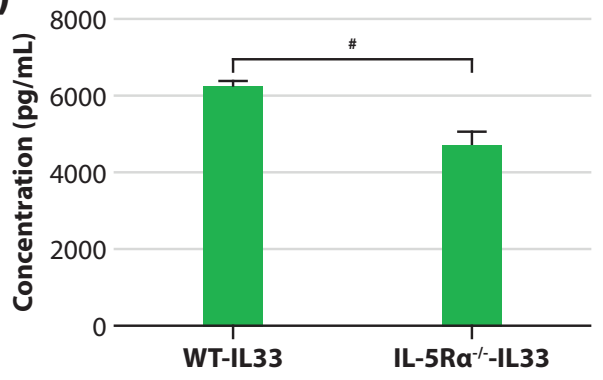

Figure 3. Evaluation of the BALF eosinophils and ILC2s in IL-33-triggered airway inflammation.

We evaluated eosinophils by karyomorphism; resident eosinophils (rEos) had a ring-shaped nucleus and inflammatory eosinophils (iEos) had a segmented nucleus (a). SiglecF+ cells were gated and analyzed as eosinophils as described in the Methods. We defined SiglecF+CD62L+ as rEos, and SiglecF+CD62L- as iEos (b). ${ }^{*}$ Comparison between the IL-33 and PBS groups $(p<0.05)$. ${ }^{\#}$ Comparison between the WT and IL-5R ${ }^{-/-}$groups $(p<0.05)$. Values are presented as the mean \pm SEM in 10 mice. The significance of differences was analyzed using 2-way ANOVA. EDN in the BALF was measured by ELISA (c). "Comparison between the WT and IL-5 $\mathrm{Ra}^{-1-}$ groups $(p<0.05)$. Values are presented as the mean \pm SEM in 20 mice. The significance of differences was analyzed using the Mann-Whitney U test. ILC2s in the BALF from WT and IL-5Ra ${ }^{-1-}$ mice were evaluated by flowcytometry (d). Lineage-negative and CD25-positive cells were defined as ILC2s as described in the Methods. "Comparison between the WT and IL-5 $\mathrm{Ra}^{-/-}$groups $(p<0.05)$. Values are presented as the mean \pm SEM in 12 mice. The significance of differences was analyzed using the Mann-Whitney U test. IL-10, IFN- $\gamma$, and TGF- $\beta_{1}$ levels were determined using Bio-Plex (e). ${ }^{*}$ Comparison between the IL-33 and PBS groups $(p<0.05)$. ${ }^{*}$ Comparison between the WT and IL-5R ${ }^{-/-}$groups $(p<0.05)$. Values are presented as the mean \pm SEM in 6 mice. The significance of differences was analyzed using 2-way ANOVA. 
(d)

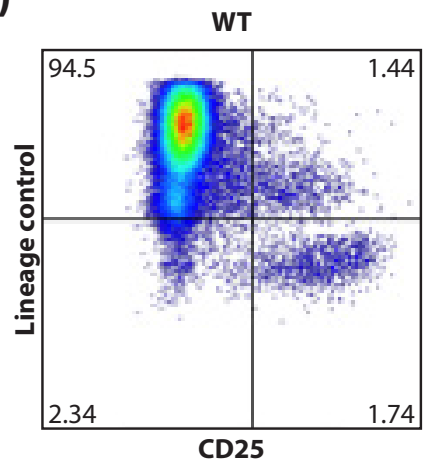

(e) 15 IL-10
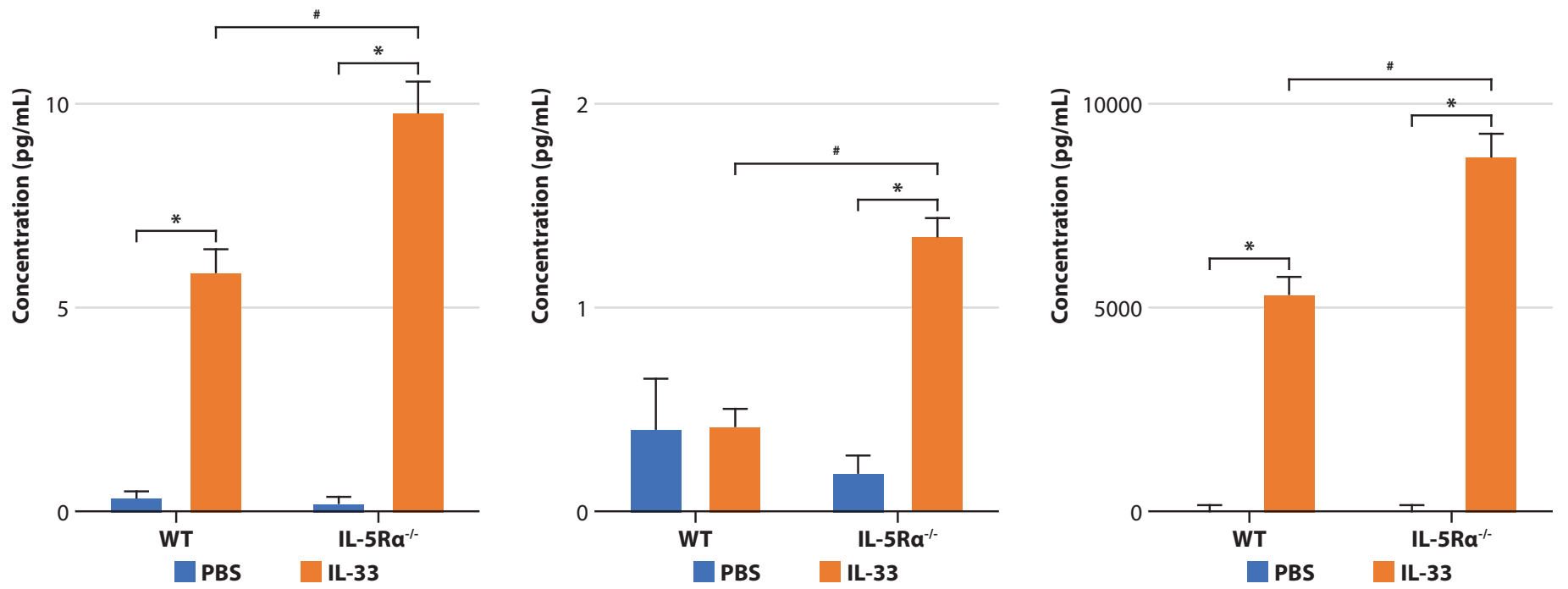
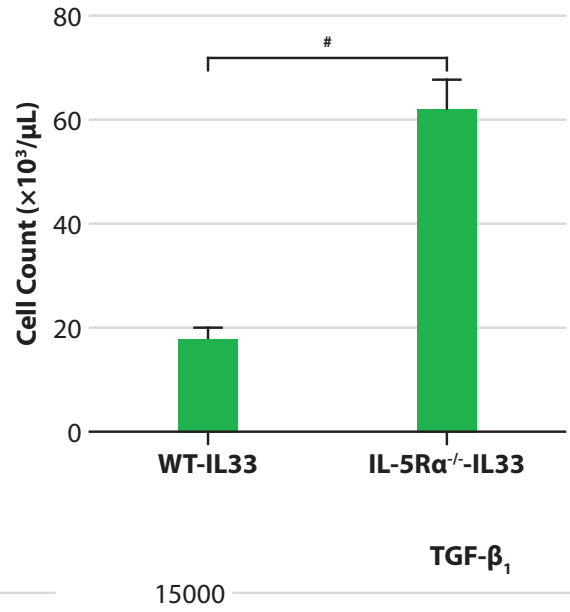

Figure 3. (Continued)

\section{Discussion}

IL-33 was identified as a functional ligand for the IL-1R family member ST2. ${ }^{8}$ ST2 is abundantly expressed on the surfaces of Th2 and ILC2. IL-33 is critical for the T2 response and can also promote the pathogenesis of asthma by inducing Th2 cells and ILC2. ${ }^{13}$ In the present study, we evaluated IL-33-triggered eosinophilic airway inflammation without an allergen in IL-5 $\mathrm{R}^{-/-}$mice. Intranasal administration of IL-33 induced T2-mediated eosinophilic airway inflammation, airway remodeling, and AHR, without increasing serum levels of IgE (data not shown) in WT mice. In IL-5 $\mathrm{Ra}^{-1-}$ mice, numbers of inflammatory cells other than eosinophils were increased in the BALF. Increased levels of IL-17 and G-CFS or MCP-1 may be involved in the accumulation of neutrophils or macrophages in the airway, respectively. It is possible that eosinophilic airway inflammation shifts toward neutrophilic airway inflammation by a complete block of IL-5 signaling in this non-allergic eosinophilic asthmatic model. AHR was found in IL-5 $\mathrm{Ra}^{-/}$mice, but levels were significantly lower than in WT mice. Histologic findings revealed that IL-33 treatment for only 3 days could induce both goblet cell hyperplasia 
compared with IL-33-treated WT mice. Interestingly, a lack of IL-5 signaling resulted in ILC2 growth and/or activation. These findings suggest that some particular negative-feedback mechanisms suppress ILC2s-mediated excessive airway inflammation in IL-33-treated mice. We evaluated cytokines that contribute to the growth, activation (IL-2, IL-4, and IL7 ), and suppression (IL-10, IFN- $\gamma$, and TGF- $\beta_{1}$ ) of ILC2s, but those results could not explain the increased number of ILC2s in the BALF of IL-5Ra ${ }^{-/-}$mice.

Recently, Mesnil et al demonstrated subpopulations of eosinophils, resident eosinophils (rEos) and inflammatory (iEos), with lung-rEos representing a distinct regulatory eosinophil subset. Mice lacking lung rEos showed increased Th2 cytokine production to inhaled allergens. ${ }^{7}$ Furthermore, recent studies of mice with genetic or anti-IL-5-mediated depletion of eosinophils showed that eosinophils are required to suppress both Th1 and Th2 responses in gastrointestinal inflammation. ${ }^{16,17}$ The findings of the present study are similar to those reported previously, ${ }^{7}$ in that we also found 2 types of eosinophils that can be distinguished by the shape of the nucleus and cell surface molecules. Furthermore, in IL-33-treated WT mice, the numbers of not only iEos but also rEos were increased in the BALF, while the numbers of both iEos and rEos were lower in the BALF of IL-33 treated IL-5 $\mathrm{Ra}^{-1-}$ mice. The rEos might suppress the excessive production of IL- 5 and IL-13 by ILC2s. Further studies are required to clarify the role of rEos in IL-33-triggered airway inflammation.

\section{Conclusion}

In the present study, in IL-5 $\mathrm{Ra}^{-/-}$mice, we found reduced eosinophilic airway inflammation, AHR, and basement membrane thickening following intranasal administration of IL-33, while excessive IL-33 induced T2 responses in the airway. The rEos induced by IL-5 might contribute to the negative feedback mechanisms in IL-33-triggered airway inflammation. Further studies are required to clarify the possible regulatory function of IL-5-induced rEos in detail.

\section{Acknowledgements}

The authors thank Ms. Tae Uesaka and Ms. Mariko Takaya for their skillful assistance. This work was supported by the Project Research Grants from Kawasaki Medical School.

\section{Disclosure Statement}

The authors declare that they have no conflicts of interest with respect to the data presented in this paper.

\section{References}

1. Busse WW, Lemanske RF Jr. Asthma. N Engl J Med. 2001;344:350-62.

2. Wills-Karp M, Karp CL. Eosinophils in athsma:remodeling a tangled tale. Science. 2004;305:1773-6.

3. Varricchi G, Bagnasco D, Borriello F, Heffler E, Canonica GW Interleukin-5 pathway inhibition in the treatment of eosinophilic respiratory disorders: evidence and unmet needs. Curr Opin Allergy Clin Immunol. 2016;16:186-200 .

4. Matsumoto N, Katoh S, Mukae H, Matsuo T, Takatsu K, Matsukura S. Critical role of IL-5 in antigen-induced pulmonary eosinophilia, but not in lymphocyte activation. Int Arch Allergy Immunol. 2003;130:209-15.

5. Shimizu H, Obase Y, Katoh S, Mouri K, Kobashi Y, Oka M. Critical role of IL-5 in the development of a mite antigen-induced chronic bronchial asthma model. Inflamm Res. 2013;62:911-7.

6. Lambrecht BN, Mammad H, Fahy JV. The cytokines of asthma. Immunity. 2021;50:975-91.

7. Mesnil C, Raulier S, Paulissen G, Xiao X, Birrell MA, Pirottin D, et al. Lung-resident eosinophils represent a distinct regulatory eosinophil subset. J Clin Invest. 2016;126:3279-95.

8. Schmitz J, Owyang A, Oldham E, Song Y, Murphy E, McClanahan TK, et al. IL-33, an interleukin-1 like cytokine that signals via the IL-1 receptor -related protein ST2 and induces T helper type 2-associated cytokines. Immunity. 2005;23:479-90 .

9. Cayrol C, Girard J-P. Interleukin-33 (IL-33):A nuclear cytokine from the IL-1 family. Immunol Rev. 2018;281:154-68.

10. Moro K, Yamada T, Tanabe M, Takeuchi T, Ikawa T, Kawamoto H et al. Innate production of $\mathrm{T}(\mathrm{H}) 2$ cytokines by adipose tissue-associated $\mathrm{cKit}(+)$ Sca-1(+) lymphoid cells. Nature. 2010;463:540-4.

11. Spits H, Artis O, Colonna M, Diefenbach A, Di Santo JP, Eberl G, et al. Innate lymphoid cells-a proposal for uniform nomenclature. Nat Rev Immunol. 2013;13:145-9.

12. Kondo Y, Yoshimoto T, Yasuda K, Futatsugi-Yumikura S, Morimoto $\mathrm{M}$, Hayashi $\mathrm{N}$, et al. Administration of IL-33 induces airway hyperresponsiveness and goblet cell hyperplasia in the lungs in the absence of adaptive immune system. Int Immunol. 2008;20:791-800.

13. Drake LY, Kita H. IL-33: Biological properties, functions and roles in airway disease. Immunol Rev. 2017;278:173-84.

14. Kabata H, Moro K, Koyasu S. The group 2 innate lymphoid cell (ILC2) regulatory network and its underlying mechanisms. Immunol Rev. 2018; 286:37-52.

15. Humbles AA, Lloyd CM, McMillan SJ, Friend DS, Xanthou G, McKenna $\mathrm{EE}$, et al. A critical role for eosinophils in allergic airways remodeling. Science. 2004;305:1776-9.

16. Strandmark J, Steinfelder S, Berek C, Kühl AA, Rausch S, Hartmann S. Eosinophils are required to suppress Th2 responses in Peyer' patches during intestinal infection by nematodes. Mucosal Immunol. 2117;10:661-72.

17. Arnold IC, Artola-Boran, M, Tallon de Lara P, Kyburz A, Taube C, Ottemann K, et al. Eosinophils suppress Th1 responses and restrict bacterially induced gastrointestinal inflammation. J Exp Med. 2018; 215:2055-72. 